\title{
Experimenting in PILAR Federation: a Common Path for the Future
}

\author{
Felix Garcia-Loro ${ }^{1}$, Alejandro Macho ${ }^{1}$, Elio San \\ Cristobal $^{1}$, Gabriel Diaz ${ }^{1}$, Manuel Castro ${ }^{1}$, Wlodek \\ Kulesza $^{2}$, Ingvar Gustavsson ${ }^{2}$, Kristian Nilsson ${ }^{2}$, \\ Andre Fidalgo $^{3}$, Gustavo Alves ${ }^{3}$, Arcelina Marques ${ }^{3}$, \\ Unai Hernandez-Jayo ${ }^{4}$, Javier Garcia-Zubia ${ }^{4}$, \\ ${ }^{1}$ Spanish University for Distance Education (UNED). \\ Madrid, Spain. \\ \{fgarcialoro; amacho, elio, gdiaz, mcastro\}@ieec.uned.es. \\ ${ }^{2}$ Blekinge Institute of Technology (BTH). \\ Karlskrona, Sweden. \\ \{wlodek.kulesza; ingvar.gustavsson; \\ kristian.nilsson\}@bth.se. \\ ${ }^{3}$ School of Engineering of Polytechnic of Porto (ISEP). \\ Porto, Portugal. \\ \{anf; gca; mmr\}@isep.ipp.pt. \\ ${ }^{4}$ University of Deusto. \\ Bibao, Spain. \\ \{unai.hernandez; zubia\}@deusto.es.
}

\begin{abstract}
The PILAR (Platform Integration of Laboratories based on the Architecture of visiR) Erasmus Plus project started in September 2016 and will last three years. The core of the PILAR project is the VISIR remote laboratory - Virtual Instruments System In Reality-. The project aims for a federation of five of the existing VISIR nodes, sharing experiments, capacity and resources among partners, and to provide access to VISIR remote lab, through PILAR consortium, to students from other educational institutions.
\end{abstract}

PILAR will be the framework from which management tasks will be performed and laboratories/experiments will be shared. PILAR will also foster the Special Interest Group of VISIR under the Global Online Laboratory Consortium (GOLC) of the International Association of Online Engineering (IAOE).

Keywords- PILAR; VISIR; federation; remote laboratory; electronics

\section{INTRODUCTION}

Practical training is extremely important in technical degree programs [1], [2]. Therefore, universities and educational institutions trust in experimentation for building successful cross-curricular learning opportunities in technical courses [3][5]. Hands-on laboratory sessions require the presence of technical and teaching personnel. It is also required an adequate room to house replicated workbenches in order to provide a suitable number of workbenches to optimize the working schedule of the aforesaid staff. So, due to these limitations, remote laboratories can play a fundamental role [6].

\author{
Christian Kreiter ${ }^{5}$, Ramona Oros ${ }^{5}$, Andreas Pester ${ }^{5}$, \\ Danilo Garbi-Zutin ${ }^{6}$, Michael Auer ${ }^{6}$, Carla Garcia- \\ Hernandez $^{7}$, Ricardo Tavio ${ }^{7}$, Kati Valtonen ${ }^{8}$, Elina \\ lehtikangas ${ }^{8}$. \\ ${ }^{5}$ Carinthia University of Applied Sciences (CUAS). \\ Villach, Austria. \\ \{c.kreiter; oros\}@fh-kaernten.at ; pester@cti.ac.at. \\ ${ }^{6}$ International Association of Online Engineering (IAOE). \\ Wien, Austria. \\ danilo@zutin.net; auer@cti.ac.at. \\ ${ }^{7}$ EVM. \\ \{carla.garcia; ricardo.tavio\}@evm.net. \\ Tenerife, Spain. \\ ${ }^{8}$ OMNIA. \\ Helsinki, Finland. \\ \{kati.valtonen; elina.lehtikangas\}@omnia.fi.
}

Remote laboratories are real laboratories —real equipment and instruments, and, if necessary, components or materialsworking on a real system under real-life conditions, controlled remotely through the Internet. The main advantage of remote laboratories, when compared to hands-on laboratories, lies on its availability that has either temporal nor geographical restrictions.

PILAR partnership is composed by 8 participants: Blekinge Institute of Technology (BTH), the origin of VISIR; University of Deusto (UDEUSTO), Spanish University for Distance Education (UNED), Carinthia University of Applied Sciences (CUAS), School of Engineering of Polytechnic of Porto (ISEP); five institutions with VISIR experiments, some of them shared, and finally, the International Association of Online Engineering (IAOE), an international non-profit organization with the objective of encouraging the wider development, distribution and application of Online Engineering; EVM has wide experience in EU projects and will co-lead and coordinate the dissemination activities; OMNIA is a multi-sector educational provider that will co-lead the training activities.

Erasmus + PILAR project aims to interconnect the partners' VISIR remote laboratories, creating a grid of shared VISIRs in order to expand and empower the circuit repository of all participants: Each institution may design certain experiments and their students perform these experiments and others installed at other institutions, and vice versa. But PILAR project is not only for institutions with a VISIR remote lab installed, it would be also possible for organization without VISIR to participate. 


\section{VISIR}

\section{A. VISIR Remote Laboratory}

VISIR remote laboratory can be divided into two blocks: The hardware block -instrumentation platform and relay switching matrix - and the software block - experiment client, measurement server and equipment server-. The equipment is shown in Fig. 1.

The instrumentation platform consists of a NI PXI chassis, where the instrument module cards (function generator, DC power supply, oscilloscope and multimeter) are connected, and an embedded (in the chassis) or external PC as a controller.

The relay switching matrix is a stack of "PCI/104" sized boards. The communication between the relay switching matrix and the PC is carried out through a USB cable. It is formed by instruments boards and components boards. Instruments boards are intended for the connection of the instrument module cards to the matrix, whereas components cards accommodate the components available for experimentation.

The VISIR software is an open-source, released under GNU General Public License (GPL). It is constituted by three modules:

- Experiment Client: the experiment client is composed by the Graphical User Interface (GUI) - flash or HTML5, depending on the version - and the Experiment Protocol (XML-based protocol). The simulated workbench is shown in Fig. 2. The users can pick components from the component tray and drag them in the holes of the virtual breadboard, wire the components, wire the equipment and measurement instruments to the components, etc. Once a user performs the experiment designed, the request is transferred to the Measurement Server using the Experiment Protocol.

- Measurement Server: Written in $\mathrm{C}++$, it is responsible

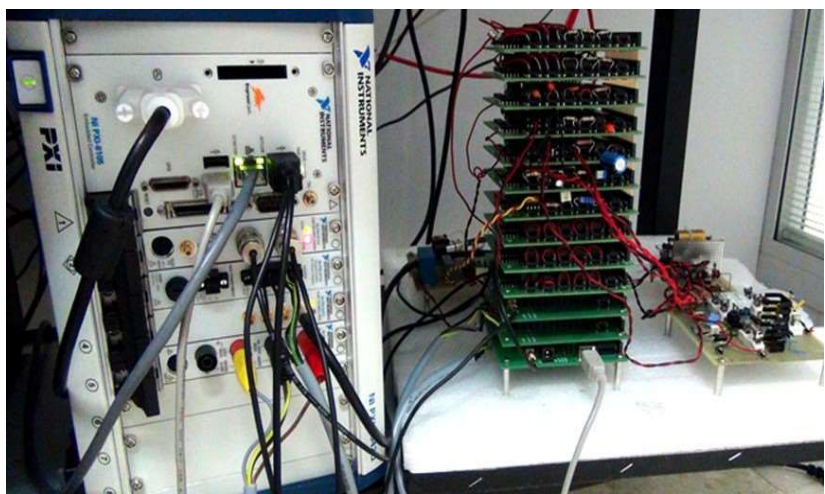

Fig. 1. PXI Chassis into which all the instrument cards (NI PXI modules) and the NI PXI controller are plugged, and relay switching matrix. VISIR system at UNED.

of receiving the request from the Experiment Client, queuing the requests from different users, checking the request (user) authentication, and verifying if the designed experiment contained in the request is allowed. Once all these steps have been completed successfully, the Measurement Server sends the request to the Equipment Server and wait to send the next one in the queue.

- Equipment Server: developed in LabVIEW, it is responsible of controlling the equipment and instruments according to the verified request received from the Measurement Server. The communication with the switching relay matrix is made through an USB cable. The NI instrument module cards are also connected to the matrix through the instrument boards.

\section{B. VISIR Reliability}

VISIR project started in 2006 at the Department of Signal Processing, BTH, in cooperation with National Instruments and

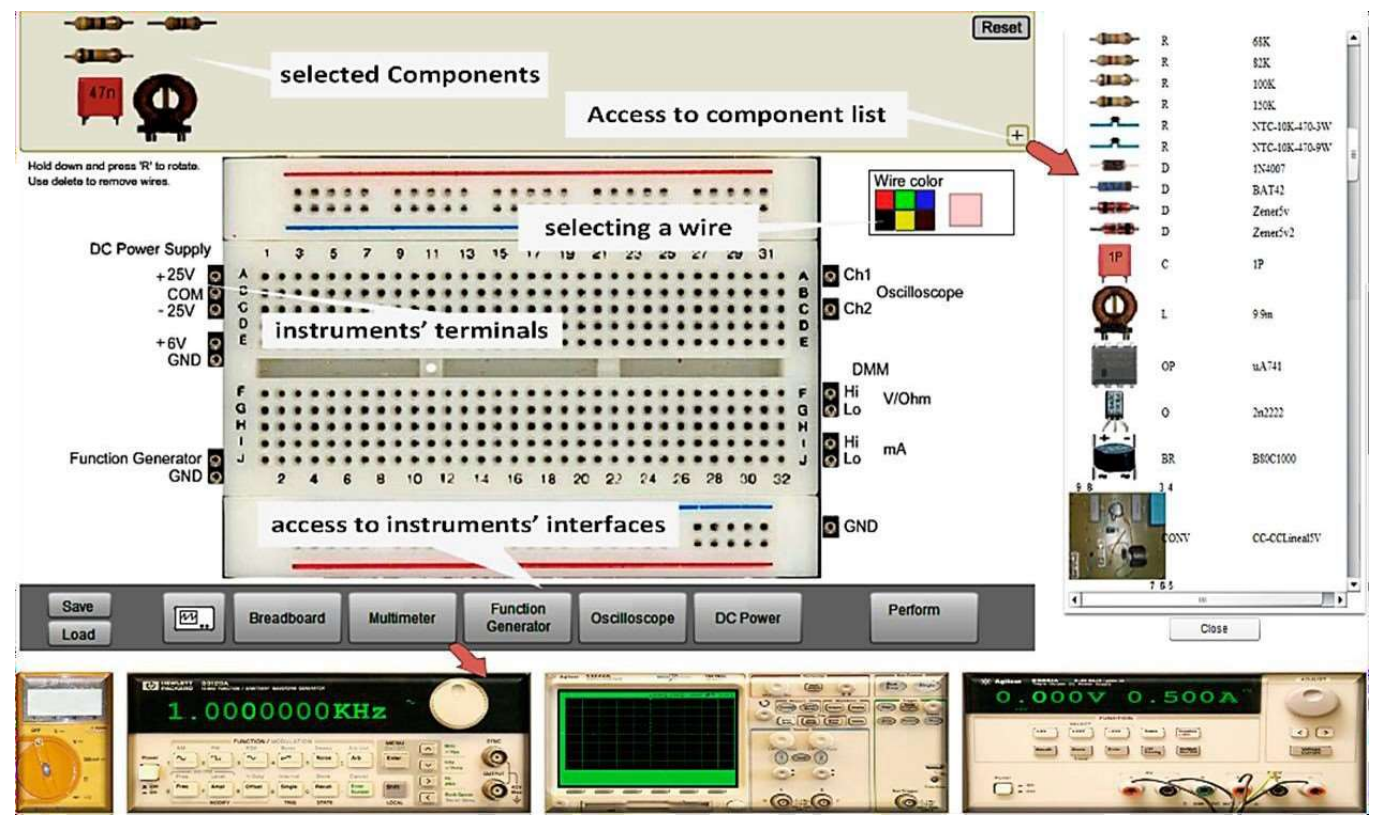

Fig. 2. Simulated workbench of VISIR (flash version). 


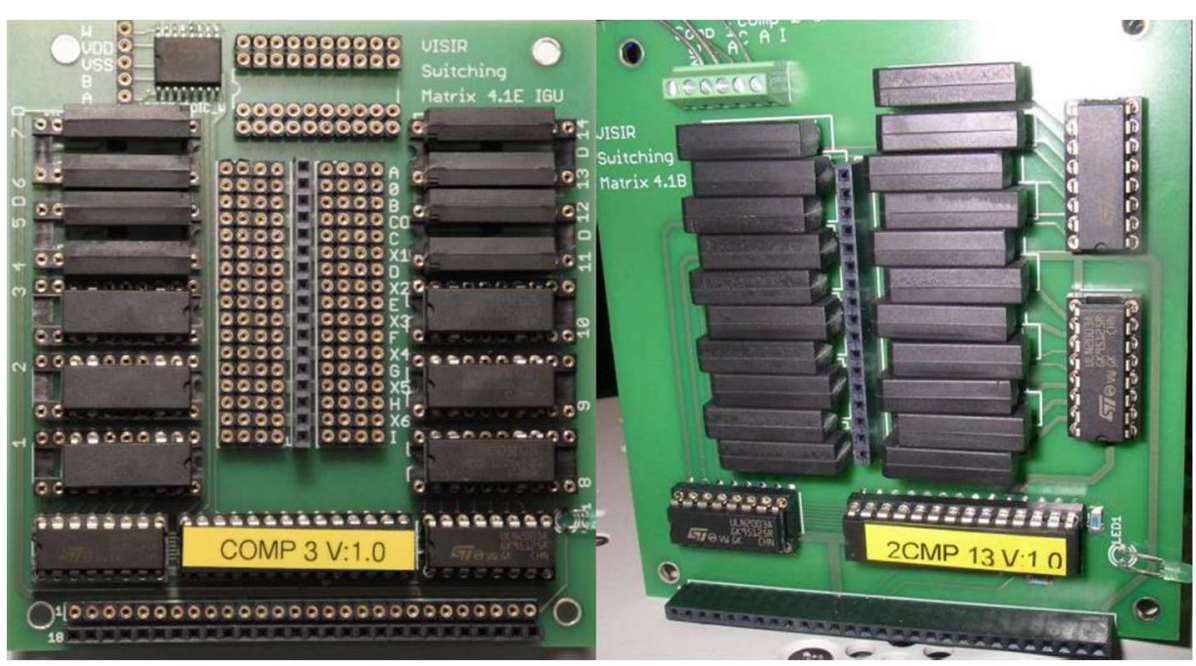

Fig. 3. Component board version E (left) and Component board 2C version B (right) [15].

Axiom EduTech and with financial support from VINNOVA (Swedish Governmental Agency for Innovation Systems) to disseminate the online laboratory concept using open source technologies [7].

So far, VISIR remote lab units are installed in thirteen different Higher Education Institutions (HEI) from eight different countries: In Argentina at National University of Rosario (UNR) and National University of Santiago del Estero (UNSE); In Austria at CUAS and FH Campus Wien for Applied Sciences; In Brazil at Federal University of Santa Catarina (UFSC), Federal Institute of Santa Catarina (IFSC) and Pontifical Catholic University of Rio de Janeiro (PUC); In Georgia at Batumi Shota Rustaveli State University (BSU); In India at Madras Institute of Technology (MIT); In Portugal at ISEP; In Spain at UDEUSTO and UNED; and in Sweden at BTH. VISIR remote lab has served well several thousands of students and has been incorporated at different educational levels and types of courses: lower secondary, upper secondary, undergraduate, postgraduate, MOOCs (Massive Open Online Courses), life-long learning, professional development, etc. [8][14].

\section{FEDERATION OF REMOTE LABS}

The federation of remote laboratories is a feature that Remote Laboratory Management Systems (RLMSs) have started to support [18], [19]. RLMSs (e.g. iLabs ${ }^{1}$ or WebLabDeusto $^{2}$ ) provide management tools and manage common services for remote laboratories [19]. Two universities using the same RLMS support federation protocols, so they can share remote laboratories to students from the "consumer" institution being the provider "university" in charge of the management tasks of their own students (e.g. authentication, authorization, etc.). Go-Lab project ${ }^{3}$ [21] counts with remote and virtual laboratories from 18 institutions.

What's more, in [20] authors describe the integration of the RLMS WebLab-Deusto as a provider for the iLab Shared
Architecture (ISA), allowing to an iLab Service Broker not only the access to the WebLab-Deusto remote labs but to share these to others iLab Service Broker and inheriting benefits from WebLab-Deusto RLMS. In this line, in [22] a federation of REMLABNET ${ }^{4}$ and Go-Lab RLMSs is introduced.

The approaches followed on these federation examples have a common factor: each remote laboratory is considered as an individual entity. In [23] authors expound the advantages of a federated system. Concerning to laboratories sharing: "once students of a particular institution can access through the Internet to a particular laboratory, it can also be accessed by students of other universities". This advantage can be bidirectional through a federation; An authors' key conclusion is that two institutions providing the same remote lab -or the same practical experience from a specific remote lab- can balance their clients/users load. This feature improves the users' involvement in the remote lab environment due to the improved time response and availability.

\section{NEED OF PILAR}

To understand the need of federate the VISIR nodes we should first understand its working process and limitations when designing circuits.

all VISIR nodes have a limitation on the available components and feasible circuits. This limitation derives from the number of component boards installed - the maximum allowable of component boards installed at each VISIR system is 15 - and the number of components installed. There are three versions of component boards:

- Component board version C: It support only ten dual pole relays. So, up to 10 components/short-cuts can be installed.

\footnotetext{
$1 \mathrm{http} / / /$ ilab.mit.edu

2 http://weblab.deusto.es/

3 www.go-lab-project.eu
}

4 www.remlabnet.eu/ 
- Component board version D/E (Fig. 3-left): they support 6 dual pole relays - intended for two-poles components - and 8 single pole relays —intended for components with more than two leads-. Any of two adjacent single pole relays can be replaced by a dual pole relay. Up to 14 components/short-cuts can be installed.

- Component board 2C version B (Fig. 3-right): Only two two-leads components can be installed in $2 \mathrm{C}$ boards, but it connects each component lead with one of five nodes.

On component boards $\mathrm{C}$ and $\mathrm{D} / \mathrm{E}$, by installing components and wiring them to the matrix nodes, it is defined how the components installed can connect the matrix - to which nodes are they connected-. Each component is conditionally connected to the matrix nodes depending on the relay control signal. The matrix nodes are from A to I and 0 (GND). The oscilloscope probes, as well as the DMM, are dynamically connected to any node. The function generator is internally connected to node A; Power sources $(6 \mathrm{~V}$; $+20 \mathrm{~V}$; $-20 \mathrm{~V})$ can be connected at any node, e.g. at UNED they are connected to nodes D, B and C respectively. A stacking connector, called node connector, propagates nodes to each component board creating a $3 \mathrm{D}$ matrix.

Fig. 4 shows the required slots for half-wave rectifier actually, it describes a half-wave rectifier comparing two diodes (1N4007 and BAT42) with different characteristics-. This experiment requires the use of 9 slots at component boards ( 2 diodes, 2 resistors and 4 short-circuits). The more complex is the circuit, the more slots it will need. Obviously, these components and short-circuits can be reused for other experiments but they will be always connected to the nodes wired at the relay switching matrix; e.g. from Fig. 4, $3.01 \mathrm{k} \Omega$ resistor wired to nodes $E$ and $F$, and $10 \mathrm{k} \Omega$ resistor wired to nodes $\mathrm{E}$ and $\mathrm{F}$ as well, can be connected in parallel (nodes $\mathrm{E}$ and F) but clearly it is impossible to connect them in series. So, the number of feasible circuits is a strong limitation for an isolated system. Furthermore, even a federation will have problems in offering all possible constructible circuits based on a relatively large set of components. However, most of these constructible circuits are worthless learning circuits or hazardous circuits for components and/or electronic

\begin{tabular}{|lll|}
\hline VFGENA_FGENA1 & A 0 & $\max : 5$ \\
VDC+6V_3 3 D & max:6 & imax:0.5 \\
VDCCOM_24_2 0 & & \\
& & \\
SHORTCUT_8_12 & F 0 & \\
R_2_8 & E F & 3K01 \\
R_2_1 & E F & $10 \mathrm{~K}$ \\
SHORTCUT_5_14 & A D & \\
SHORTCUT_1_12 & C D & \\
D_3_1 & C E & 1N4007A \\
SHORTCUT_2_5 & B C & \\
D_3_8 & C E & BAT42 \\
SHORTCUT_6_12 & A F & \\
\hline
\end{tabular}

Fig. 4. File describing the available conections in the relay switching matrix. The described components and shortcircuits must be implemented at the component boards.

equipment/instruments.

A federation of VISIR systems will not only provide a substantial circuit repository and variations based on their components but also an optimization of component and time resources at each node. Fig. 5-left shows a component box used by students in Electronics Engineering Fundamentals hands-on laboratory sessions at UNED. There are 8 exact replicas of it -one for each workbench - and another box for spare components (burned components, leads broken, fuses for DMMs, etc.). Along this line, Fig. 5-right shows the electronic components store for the subjects with practical work related to electrical/electronics at ISEP. While, to install all the components and design the circuits in the VISIR matrix required for Electronics Engineering Fundamentals practices at UNED, Fig. 5-left, involve over 5 component boards (version $\mathrm{D} / \mathrm{E}$ ) but no replicas are needed as all students will share the same components. Additionally, neither components nor equipment/instruments are damaged due to the software protection layer. However, its permanent installation would restrict VISIR use for other subjects. The difference between a switching matrix storage capacity (around 70 components for 10 component boards version $\mathrm{D} / \mathrm{E}$ ) and a typical electronic components store (Fig. 5-right) highlights the needs of a federation.

Another limitation is the number of concurrent users, no more than 60 students can experiment at a VISIR system, furthermore, as exposed in [16], the increasing delay in managing the requests/responses for an increasing number of

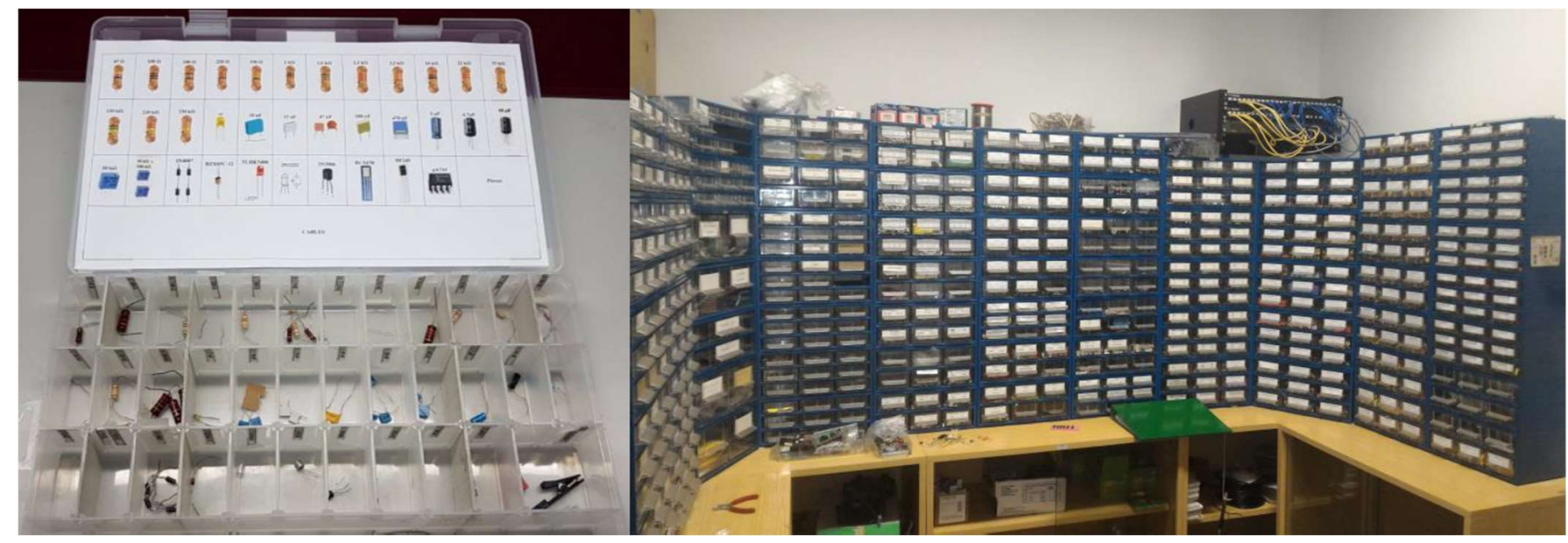

Fig. 5. Laboratory component box for Electronics Engineering Fundamentals at DIEEC-UNED (left); And the components store at ISEP-IPP. 
simultaneous users by an "isolated" VISIR system, helps to understand the capability of a federation.

By creating a grid of shared VISIRs, each partner will increase and empower their own practices repository for students and teachers. As stated in [17] "this situation will push innovation and collaboration between nodes and students, and entities will be able to manage and control students' learning process in a much better and easier way".

\section{FEDERATION APPROACHES}

One requirement of the PILAR project is the reliability of VISIR remote lab services offered through the Internet. Although a federation provides a key factor to overcome reliability premise: redundancy, Availability Metrics were designed - as a part of PILAR Intellectual Output 3 (IO3) «VISIR State of the Art Report»- in order to evaluate the VISIR systems availability over time in each node. They are divided in two main categories, to better quantify and discriminate results:

- Operational Availability is used to measure the percentage of days the VISIR system was available to students, since installation on each institution. This metric quantifies the actual number of days VISIR system was made available for usage on experiments as part of a planned course curricula.

- System Availability is used to measure the ratio of time the system was up when it should be available and it quantifies the shortages due to technical or logistical issues.

These metrics were evaluated on all partners and both were rated as very high. Results are not based on factual metrics, as these are not recorded in the present system in none of the institutions. Available metrics are based on estimates by technical and management personnel. VISIR system is active between 6 and 10 months yearly, depending on the node, providing practical support to several courses and educational levels [8]-[14].

The Operational Availability average was over 95\%, with only UNED stating that during some period - 2016 - there was a $50 \%$ availability due to for system reconfiguration, when it was not being used by students. In general, VISIR systems have been available when required on all institutions with no cases of unwanted unavailability.

As to System Availability, the average is over $90 \%$, slightly lower as some issues did occur that required action. Usual issues were network constraints or electrical power outages, usually external to VISIR system. The problems were normally solved quickly, the reason for the lower estimate and consequent delays being, sometimes, the long time required for staff to be made aware of the problem.

Since the origin of VISIR, it has been improved continuously (both, hardware and software); new versions of component and instrument boards have been released, and the software layer has been upgraded several times. This continuous improvement together with the Availability Metrics results confirm that VISIR is a robust and reliable system.
The analysis carried out in Intellectual Output 2 (IO2) has been summarized in a document describing the different kind of practices, its usage and training levels offered by VISIR nodes. The aforementioned document has served to propose how to improve technically the different VISIR nodes in the federation, how to complement the different catalogs and how to stablish more pedagogical practices for the different education levels. Preliminary conclusions of this section were:

- All experiments have guidance or learning material developed as support material for users.

- The main fields are: electricity, basic analog electronics, electronics and power electronics, and physics.

- Basic electronics experiments are available on every VISIR system, all partners have developed similar experiments Kirchhoff's laws, Ohm's law, RL circuit, teaching transistors, operational amplifiers, passive filters, etc.

- The education level on which the experiments are used: high school (15 - 18/19), bachelor, master.

The aforementioned document has allowed to identify practical experience gaps, key and common topics or new possibilities. Regarding the experiments designed in the switching relay matrices, 4 approaches are being analyzed:

- Redundancy approach. This approach empowers scalability, reliability and availability at the expense of diversity. If one node is not available for any reason, its effort can be supported by other nodes. That is: if one user is accessing the VISIR system in his institution but it is down, she/he will be automatically redirected to another available VISIR node, allowing swiftly routing the clients' requests to whichever node can respond. In this case, the federation mechanism must identify those redundant experiments.

- Diversity approach. each VISIR node can specialize in a specific topic (taking advantage of reusability) and implement a set of circuits (DC circuits, AC circuits, OAs circuits, transistors, etc.). The user will not access only to the set of experiments of his institution, but also to the total set of experiments of all the VISIR nodes. This approach empowers experiment catalog at the expense of scalability and reliability.

- Combined approach. Using a combination of both above approaches, empowering scalability for those common experiments and specializing each node in a specific area.

- $\quad$ Agreed-Shared approach. Each node has to design a percentage of their components boards based on the federation needs (redundancy, diversity or combined), and design freely the rest of component boards sharing the experiments with the partners.

\section{CONCLUSIONS AND FUTURE WORK}

It is visible that partners feel the need to develop experiments concerning topics which are already available on other partners systems. The main finding underlines, even 
more, the need of a VISIR federation and the idea of sharing experiments.

Any of the approaches suggested for the experiments federation in PILAR will improve the efficiency of each VISIR system already built and will allow partners to share their learnings and capabilities of their respective VISIR systems and experiences with the rest of the academic community.

As future work, the architecture of the Federation will be established soon, as it is being technically planned nowadays. Once it will be defined, a pilot experience will be released as part of Work Package 2. The main challenge is derived from the different architectures implemented at each node. BTH and ISEP use OpenLabs ${ }^{5}$ platform, CUAS have iLabs as RLMS, whereas WebLab-Deusto is the RLMS at UNED and UDEUSTO. This pilot federation and the results obtained from it will be applied in a "Plan-Do-Check-Act" Deming cycle.

\section{ACKNOWLEDGMENT}

The authors acknowledge the support of the PR-VISIR, PIE13, "Prácticas Remotas de Electrónica en la UNED, Europa y Latinoamérica con Visir", Escuela de Doctorado de la UNED, eMadrid project (Investigación y Desarrollo de Tecnologías Educativas en la Comunidad de Madrid) - S2013/ICE-2715, VISIR+ project (Educational Modules for Electric and Electronic Circuits Theory and Practice following an Enquirybased Teaching and Learning Methodology supported by VISIR) Erasmus + Capacity Building in Higher Education 2015 n ${ }^{\circ}$ 561735-EPP-1-2015-1-PT-EPPKA2-CBHE-JP and PILAR project (Platform Integration of Laboratories based on the Architecture of visiR), Erasmus + Strategic Partnership $n^{\circ}$ 2016-1-ES01-KA203-025327,. MECA - MicroElectronics Cloud Alliance - Erasmus + Knowledge Alliances $2015 \mathrm{n}^{\mathrm{o}}$ 562206-EPP-1-2015-1-BG-EPPKA2-KA.

\section{REFERENCES}

[1] J. Rodriguez-Andina, L. Gomes, and S. Bogosyan. "Current Trends in Industrial Electronics Education", IEEE Transactions on Industrial Electronics, vol. 57, no. 10, pp. 3245-3252, 2010.

[2] Using Remote Labs in Education: Two Little Ducks in Remote Experimentation. Editors: Javier Garcia- Zubia (Universidad de Deusto) and Gustavo R. Alves (Polytechnic of Porto). Universidad de Deusto, Bilbao, 2011. 22 chapters. 465 pp. ISBN 978-84-9830-335-3.

[3] O. Naef. Real Laboratory, virtual laboratory or remote laboratory: what is the most ef-fective way?. Intl. Journal of Online Engineering, Vol 2, No.3. (2006)

[4] Z. Nedic, J. Machotka, and A. Nafalski: Remote laboratories versus virtual and real laboratories, 34th ASEE/IEEE frontiers in education conference, session T3E-1, pp.1-6. November 2003 .

[5] A. Coble, A. Smallbone, A. Bhave, R. Watson, A. Braumann, and M. Kraft. Deliver-ing authentic experiences for engineering students and professionals through e-labs. IEEE EDUCON. pp 1085 - 1090. (2010).

[6] L. Gomes and S. Bogosyan. "Current Trends in Remote Laboratories", IEEE Transactions on Industrial Electronics, vol. 56, no. 12, pp. 47444756, 2009.

[7] I. Gustavsson, J. Zackrisson, L. Håkansson, I. Claesson, T. Lagö, “The VISIR project - an open source software initiative for distributed online laboratories," International Conference on Remote Engineering and Virtual Instrumentation (REV), 2007, Porto.
[8] I. Gustavsson, K. Nilsson, J. Zackrisson, G. Alves, A. Fidalgo, L. Claesson, J. Zubia, U. Jayo, M. Castro, G. Diaz Orueta and F. Loro, "Lab sessions in VISIR laboratories", 2016 13th International Conference on Remote Engineering and Virtual Instrumentation (REV), 2016.

[9] M. Marques, M. Viegas, M. Costa-Lobo, A. Fidalgo, G. Alves, J. Rocha and I. Gustavsson, "How Remote Labs Impact on Course Outcomes: Various Practices Using VISIR", IEEE Transactions on Education, vol. 57, no. 3, pp. 151-159, 2014.

[10] F. Garcia, G. Diaz, M. Tawfik, S. Martin, E. Sancristobal and M. Castro, "A practice-based MOOC for learning electronics", 2014 IEEE Global Engineering Education Conference (EDUCON), 2014.

[11] A. Fidalgo, G. Alves, M. Marques, M. Viegas, M. Costa-Lobo, U. Henandez-Jayo, J. Garcia-Zubia and I. Gustavsson, "Adapting Remote Labs to Learning Scenarios: Case Studies Using VISIR and RemotElectLab", IEEE Revista Iberoamericana de Tecnologias del Aprendizaje, vol. 9, no. 1, pp. 33-39,2014.

[12] M. Tawfik, S. Monteso, F. Loro, E. Sancristobal, F. Mur, G. Diaz and M. Castro, "Design of electronics circuits practices for an online master degree program using VISIR", 2013 IEEE Global Engineering Education Conference (EDUCON), 2013.

[13] S. Marchisio, F. Lerro, C. Merendino, M. Plano, S. Concari, G. de Arregui, J. Garcia-Zubia, U. Hernandez-Jayo, G. Alves and I. Gustavsson, "Starting the study of electronic circuits with VISIR: Viewpoints of college students in a pilot test in Argentina", 2017 4th Experiment@International Conference (exp.at'17),2017.

[14] Natércia Lima, Clara Viegas, Gustavo Alves and Francisco J. GarcíaPeñalvo; "VISIR's Usage as a Learning Resource: a Review of the Empirical Research", 4th Technological Ecosystems for Enhancing Multiculturality (TEEM'16), Salamanca, Spain, November 2-4, 2016.

[15] I. Gustavsson, Visir Relay Switching Matrix Version 4.1 - User's Manual (ver.7). 2016.

[16] G. Alves et al., "Spreading remote lab usage a system - A community - A Federation", 2nd International Conference of the Portuguese Society for Engineering Education (CISPEE), 2016.

[17] W. Kulesza et al., "A federation of VISIR remote laboratories through the PILAR Project", 4th Experiment@International Conference (exp.at'17), 2017.

[18] V. J. Harward, et al., "The ilab shared architecture: A web services infrastructure to build communities of internet accessible laboratories,"Proceedings of the IEEE, vol. 96, no. 6, pp. 931-950, 2008.

[19] P. Orduna et al., "Generic integration of remote laboratories in learning and content management systems through federation protocols", 2013 IEEE Frontiers in Education Conference (FIE), 2013.

[20] P. Orduna, J. Garcia-Zubia, D. Lopez-de-Ipina, P. Bailey, J. Hardison, K. DeLong and V. Harward, "Sharing Laboratories across Different Remote Laboratory Systems", 2012 IEEE 12th International Conference on Advanced Learning Technologies, 2012.

[21] Go-Lab, European Commission-CORDIS: Projects and Results: Global Online Science Labs for Inquiry Learning at School", Cordis.europa.eu, 2018. [Online]. Available: http://cordis.europa.eu/project/rcn/105919_en.html. [Accessed: 12- Jan2018].

[22] F. Schauer, M. Krbecek, P. Beno, M. Gerza, L. Palka, P. Spilakova and L. Tkac, "REMLABNET III - Federated remote laboratory management system for university and secondary schools", 2016 13th International Conference on Remote Engineering and Virtual Instrumentation (REV), 2016.

[23] P. Orduña, P. Bailey, K. DeLong, D. López-de-Ipiña and J. GarcíaZubia, "Towards federated interoperable bridges for sharing educational remote laboratories", Computers in Human Behavior, vol. 30, pp. 389$395,2014$.

\footnotetext{
${ }^{5}$ http://openlabs.bth.se/
} 
